PROCEEDINGS OF THE

AMERICAN MATHEMATICAL SOCIETY

Volume 45, Number 2, August 1974

\title{
STEINHAUS TYPE THEOREMS FOR SUMMABILITY MATRICES
}

\author{
I. J. MADDOX
}

ABSTRACT. Necessary and sufficient conditions are given for an infinite matrix to sum all bounded strongly summable sequences. It is shown that the Borel matrix does not sum all such sequences. A corollary is that the bounded summability field of the Borel method is strictly contained in that of the $(C, 1)$ method. Also, it is proved that no coregular matrix can almost sum all bounded sequences-a generalization of Steinhaus' theorem.

1. Introduction. The well-known theorem of Steinhaus asserts that no Toeplitz matrix can sum all bounded sequences. A similar type of result due to Kuttner [4] states that, for $0<p<1$, no Toeplitz matrix can sum all sequences in $w_{p}$ (the space of all strongly $(C, 1)$ summable sequences with index $p$ ). These theorems of Steinhaus and Kuttner are also valid if Toeplitz matrices are replaced by coregular matrices [6].

It may be observed that the sequence in [4], which is in $w_{p}$ but is not summable by the Toeplitz matrix, is unbounded. Consequently it is natural to consider whether or not a Toeplitz matrix can sum all bounded sequences in $w_{p}$. It is easy to see that there are Toeplitz matrices which do sum all bounded sequences in $w_{p}$. In fact if $p>0$ and $r>0$ then it is well known that, for bounded sequences, $w_{p}$ is equal to $w_{1}$ and $(C, r)$ is equivalent to $(C, 1)$. Hence, if $l_{\infty}$ denotes the space of bounded sequences, then $l_{\infty} \cap$ $w_{p} \subset l_{\infty} \cap(C, r)$, so that the Toeplitz matrix of $(C, r)$ means sum every sequence in $l_{\infty} \cap w_{p}$. Obviously the unit matrix does not sum every sequence in $l_{\infty} \cap w_{p}$, and it is easy to see that there are Toeplitz matrices not equivalent to convergence which do not sum every sequence in $l_{\infty} \cap w_{p}$. These observations lead us to characterize (in $\$ 3$ ) all infinite matrices which sum every sequence in $l_{\infty} \cap w_{p}$.

Received by the editors October 1, 1973.

AMS (MOS) subject classifications (1970). Primary 40C05, 40D20; Secondary 40G05, 40G10.

Key words and phrases. Bounded strongly summable sequences, infinite matrix, conservative matrix, Cesàro means, Borel matrix, summability, almost summability. 
In $\$ 4$ we consider Steinhaus type theorems in which summability by a matrix is replaced by 'almost summability'.

2. Definitions. By $l_{\infty}$ we denote the Banach space of all bounded complex sequences $x=\left(x_{k}\right)$ with $\|x\|=\sup \left|x_{k}\right|$. By $c$ and $f$ we denote the closed subspaces of convergent and almost convergent sequences. The almost convergent sequences $f$ were introduced by Lorentz [5] via Banach limits. With strict inclusion we have $c \subset f \subset l_{\infty}$.

For $p>0$ we define $w_{p}$ to be the space of all $x$ such that, for some number $l$,

$$
\frac{1}{n} \sum_{k=1}^{n}\left|x_{k}-l\right|^{p} \rightarrow 0 \quad(n \rightarrow \infty) .
$$

The space $w_{p}$ has been considered by several authors [1], [4], [6].

If $A=\left(a_{n k}\right)$ is an infinite matrix of complex numbers we write $A_{n}(x)=$ $\sum a_{n k} x_{k}$, all sums being over $1 \leq k \leq \infty$, unless otherwise indicated. We write $A x=\left(A_{n}(x)\right)$.

For nonempty sets of sequences $X, Y$ we denote by $(X, Y)$ the set of all $A$ such that $A x \in Y$ for every $x \in X$. Also, we define $X(A)=\{x: A x \in X\}$, so for example $c(A)$ is the summability field of $A$, and $f(A)$ is the set of all $x$ which are almost summable by $A$. Some results on almost summability may be found in [3].

It is familiar that $A \in(c, c)$ if and only if $\|A\|=\sup _{n} \Sigma\left|a_{n k}\right|<\infty$, $\lim _{n} a_{n k}=a_{k}($ each $k), \lim _{n} \Sigma a_{n k}=a$. The space $(c, c)$ of all conservative matrices is a Banach algebra under the matrix product, and the characteristic $\chi$ defined by $\chi(A)=a-\Sigma a_{k}$ is a scalar homomorphism on $(c, c)$. The kernel of $\chi$ is written $K_{0}$ and is called the conull matrices. The set $(c, c)-$ $K_{0}$ of coregular matrices is written as $K$.

Finally, a set $E$ of positive integers is said to be of zero density if the number of elements of $E$ which lie in $[1, n]$ is $o(n)$ as $n \rightarrow \infty$. For example, $\left\{2,2^{2}, 2^{3}, \cdots\right\}$ is of zero density.

3. Summability of bounded strongly summable sequences.

Theorem 1. Let $p>0$. Then $A \in\left(l_{\infty} \cap w_{p}, c\right)$ if and only if $A$ is conservative and

$$
\sum_{k \in E}\left|a_{n k}-a_{k}\right| \rightarrow 0 \quad(n \rightarrow \infty)
$$

for each set $E$ of zero density.

Proof. For the sufficiency let $x \in l_{\infty}$ and suppose (1) holds. Since $A$ is conservative it is sufficient to show that 


$$
\sum\left|a_{n k}-a_{k}\right| \cdot\left|x_{k}-l\right| \rightarrow 0 \quad(n \rightarrow \infty) .
$$

Let $\epsilon>0$ and write $E=\left\{k:\left|x_{k}-l\right| \geq \epsilon\right\}$. Since $x_{k} \rightarrow l\left(w_{1}\right)$ it is clear that $E$ has zero density. Hence, for all $n$,

$$
\begin{array}{r}
\sum_{k \in E}\left|a_{n k}-a_{k}\right| \cdot\left|x_{k}-l\right|+\sum_{k \in-E}\left|a_{n k}-a_{k}\right| \cdot\left|x_{k}-l\right| \\
\leq 2\|x\| \cdot \sum_{k \in E}\left|a_{n k}-a_{k}\right|+2 \epsilon\|A\| \cdot .
\end{array}
$$

Now letting $n \rightarrow \infty$ we see that (3) holds.

Consider now the necessity. From $A \in\left(l_{\infty} \cap w_{p}, c\right)$ and the fact that $c \subset l_{\infty} \cap w_{p}$ it follows that $A \in(c, c)$. Suppose, if possible, that there exists a set $E$ of zero density such that (2) fails. This implies that $E$ is an infinite set, so we write $E=\left\{e_{1}, e_{2}, \cdots\right\}$. By the proof of Schur's theorem [7, p. 169] there is a bounded sequence $z=\left(z_{e_{1}}, z_{e_{2}}, \cdots\right)$ such that the sequence $\left(\Sigma_{k \in E}\left(a_{n k}-a_{k}\right) z_{k}\right)$ is divergent. Now define a sequence $x$ by $x_{k}=$ $z_{k}$ for $k=e_{i}$ and $x_{k}=0$ for $k \neq e_{i}$. Then $x \in l_{\infty}$, and since $E$ has zero density we see that $x_{k} \rightarrow 0\left(w_{p}\right)$. Hence there exists $x \in l_{\infty} \cap w_{p}$ such that $x \notin$ $c(A)$, which is contrary to $A \in\left(l_{\infty} \cap w_{p}, c\right)$. This proves the theorem.

As an application of Theorem 1 we consider the Borel matrix defined by $a_{n k}=e^{-n} n^{k} / k !$, with $n, k \geq 0$.

Theorem 2. The Borel matrix does not sum all sequences in $l_{\infty} \cap w_{p}$.

Proof. Define $E=\left\{e_{1}, e_{2}, \cdots\right\}$, where $e_{k}=4^{i}+k-2^{i}$ for $2^{i} \leq k<$ $2^{i+1}$ and $i=0,1,2, \cdots$ Thus $E=\{1,4,5,16,17,18,19,64,65, \ldots\}$. Now take $n \geq 1$ and determine $i$ such that $4^{i} \leq n<4^{i+1}$. Then the number of elements of $E$ which lie in $[1, n]$ is less than or equal to $1+2+\cdots$ $+2^{i}<2 n^{1 / 2}=o(n)$, so that $E$ is of zero density. Now let $\left(a_{n k}\right)$ be the Borel matrix, and write $m=4^{n}$. Then

$$
\sum_{k \in E}\left|a_{m k}\right|>\sum_{b=0}^{m^{1 / 2}-1} a_{m, m+b} \text {. }
$$

Using the simple inequality $p ! \leq e^{-p+1} p^{p} p^{1 / 2}$, valid for positive integers $p$, we find that

$$
\begin{aligned}
\log a_{m, m+b} \geq & -m+(m+b) \log m-(m+b) \log (m+b) \\
& -\log (m+b)^{1 / 2}+m+b-1 \\
= & -(m+b) \log (1+h / m)-\log (m+b)^{1 / 2}+b-1 \\
\geq & -b-h^{2} / m-\log (2 m)^{1 / 2}+b-1,
\end{aligned}
$$


whence

$$
a_{m, m+b} \geq \exp \left(-b^{2} / m\right) / e(2 m)^{1 / 2} \text { for } 0 \leq b<m^{1 / 2} .
$$

By (4) it now follows that

$$
\sum_{k \in E}\left|a_{m k}\right|>\frac{1}{e(2 m)^{1 / 2}} \int_{0}^{m^{1 / 2}} e^{-t^{2} / m} d t=\frac{1}{e 8^{1 / 2}} \int_{0}^{1} e^{-u} u^{-1 / 2} d u \text {, }
$$

which implies that $\lim \sup _{n} \Sigma_{k \in E}\left|a_{n k}\right|>0$. By Theorem 1 it follows that $A \notin\left(l_{\infty} \cap w_{p}, c\right)$.

An immediate consequence of Theorem 2 is that if $S$ is any summability method which is included in the Borel method then $S \notin\left(l_{\infty} \cap w_{p}, c\right)$. In particular this applies when $S=(E, q)$, the Euler method [2, Theorem 128]. Another corollary to Theorem 2 is that the bounded summability field of the Borel method is strictly contained in the bounded summability field of the $(C, 1)$ method. To see this we write $A_{n}=a_{0}+a_{1}+\cdots+a_{n}$ and assume that $\left(A_{n}\right)$ is bounded and Borel summable. Hence $a_{n}=o(n)$, so by [2, Theorem 147], with $\rho=1$, we have that $\left(A_{n}\right)$ is $(C, 3)$ summable, and so $(C, 1)$ summable since $\left(A_{n}\right)$ is bounded. Thus we have $l_{\infty} \cap$ Borel $\subset l_{\infty} \cap(C, 1)$. If equality occurred in this last inclusion then we would obtain a contradiction by Theorem 2.

4. Almost summability of bounded sequences. Steinhaus' theorem may be put in the following form: if $A$ is coregular then the inclusion $l_{\infty} \subset c(A)$ is false. Now for any matrix $A$ we have $c(A) \subset f(A)$, so that it seems possible that the inclusion $l_{\infty} \subset f(A)$ might be true for coregular matrices. However, in Theorem 3 (iii) we show otherwise. Consequently, we have a generalization of Steinhaus' theorem, viz. that no coregular matrix can almost sum all bounded sequences.

Theorem 3. Let $A$ and $B$ be conservative matrices and suppose that $A \in\left(l_{\infty}, c(B)\right)$. Then

(i) $B A \in K_{0}$.

(ii) If $B \in K$ then $A \in K_{0}$.

(iii) If $A \in K$ then $l_{\infty} \subset f(A)$ is false.

Proof. (i) Since $A \in\left(l_{\infty}, c(B)\right)$ we have $B(A x) \in c$ for all $x \in l_{\infty}$. Now $A$ and $B$ conservative implies $B(A x)=(B A) x$ for all $x \in l_{\infty}$, whence $(B A) x \in c$ for all $x \in l_{\infty}$, so that $B A \in\left(l_{\infty}, c\right) \subset K_{0}$.

(ii) $\mathrm{By}(\mathrm{i})$ and the fact that $\chi$ is a scalar homomorphism we have $\chi(B) \chi(A)=0$, whence the result.

(iii) If $A \in K$ but $l_{\infty} \subset f(A)$, then $A \in\left(l_{\infty}, f\right)$ which implies $A \in\left(l_{\infty}\right.$, $(C, 1)$ ). Hence, by (ii), on taking $B$ to be the coregular $(C, 1)$ matrix we obtain $A \in K_{0}$, contrary to $A \in K$. 


\section{BIBLIOGRAPHY}

1. D. Borwein, Linear functionals connected with strong Cesàro summability, J. London Math. Soc. 40 (1965), 628-634. MR 32 \#2893.

2. G. H. Hardy, Divergent series, Clarendon Press, Oxford, 1949. MR 11, 25.

3. J. P. King, Almost summable sequences, Proc. Amer. Math. Soc. 17 (1966), 1219-1225. MR 34 \#1752.

4. B. Kuttner, Note on strong summability, J. London Math. Soc. 21 (1946), 118-122. MR 8, 375 .

5. G. G. Lorentz, A contribution to the theory of divergent series, Acta Math. 80 (1948), 167-190. MR 10, 367.

6. I. J. Maddox, On Kuttner's theorem, J. London Math. Soc. 43 (1968), 285-290. MR $37 \# 641$.

7. Elements of functional analysis, Cambridge Univ. Press, New York, 1970.

DEPARTMENT OF PURE MATHEMATICS, QUEEN'S UNIVERSITY OF BELFAST, NORTHERN IREL AND 